\title{
A Surgical Technique for Optic Pit Maculopathy: Vitrectomy Combined with Reversed ILM Flap
}

\author{
Abdullah Ozkaya, Gurkan Erdogan \\ Beyoglu Eye Training and Research Hospital, Istanbul, Turkey
}

\begin{abstract}
This report is a description of a surgical technique for optic disc maculopathy (OPM). Pars plana vitrectomy combined with internal limiting membrane (ILM) peeling, reverse ILM flap insertion into optic pit, and gas tamponade surgery was performed on 5 patients with OPM. Preliminary outcomes of the technique were promising.

Keywords: Internal limiting membrane, optic pit, vitrectomy
\end{abstract}

\section{Introduction}

Optic disc pits (ODPs) were first described by Wiethe in $188 \mathrm{I}$ and ascribed to incomplete fetal closure of the optic nerve (I). They are usually asymptomatic in their uncomplicated forms. However, in $25 \%$ to $75 \%$ of patients, optic pit maculopathy (OPM), which usually causes visual deterioration, may be detected. Optic maculopathy is characterized by serous retinal detachment, usually at the posterior pole. Sometimes cystic retinal changes may accompany serous retinal detachment. Origin of subretinal fluid in OPM is controversial. Four different sources have been proposed: vitreous fluid, cerebrospinal fluid, fluid from leaky blood vessels at the base of the pit, and fluid originating from the orbital space surrounding the dura. Although ODP is congenital, OPM is usually reported at around 30 years of age. No treatment modality has been universally accepted in OPM cases. Conservative management was used initially, which resulted in $25 \%$ resolution; however, visual outcomes were very poor. Several treatment options were then described. Bed rest with bilateral patching and oral corticosteroids; laser photocoagulation; intravitreal gas injection; macular buckling sur- gery; pars plana vitrectomy (PPV), with or without internal limiting membrane (ILM) peeling; and many other adjuvants to PPV. In this surgical technique report, PPV combined with ILM peeling, reverse ILM flap insertion into optic pit, and gas tamponade surgery in patients with OPM is described $(2,3)$.

\section{Surgical Technique}

Under general or subtenon anesthesia (according to patient characteristics), standard, 3-port, 23-gauge scleral incisions $3.5 \mathrm{~mm}$ from the limbus were performed. First, core vitrectomy was performed, triamcinolone-assisted posterior vitreous detachment was induced, and then posterior vitrectomy was completed. Fluid-air exchange was performed for ILM staining with $0.25 \%$ Brilliant Blue G. Dye was aspirated with flute cannula and air-fluid exchange was completed. First puncture to ILM was made with ILM micro forceps near the optic pit cavity to create ILM flap that would cover the cavity (Figure Ia). ILM flap was released from areas adjacent to the optic pit and left in place (Figure Ib). Large area of ILM was peeled, extending to at least 2-3 disc diameters, in order to cover location of serous retinal detachment (Figure Ic). Prepared ILM flap was shortened with vitreous cutter 

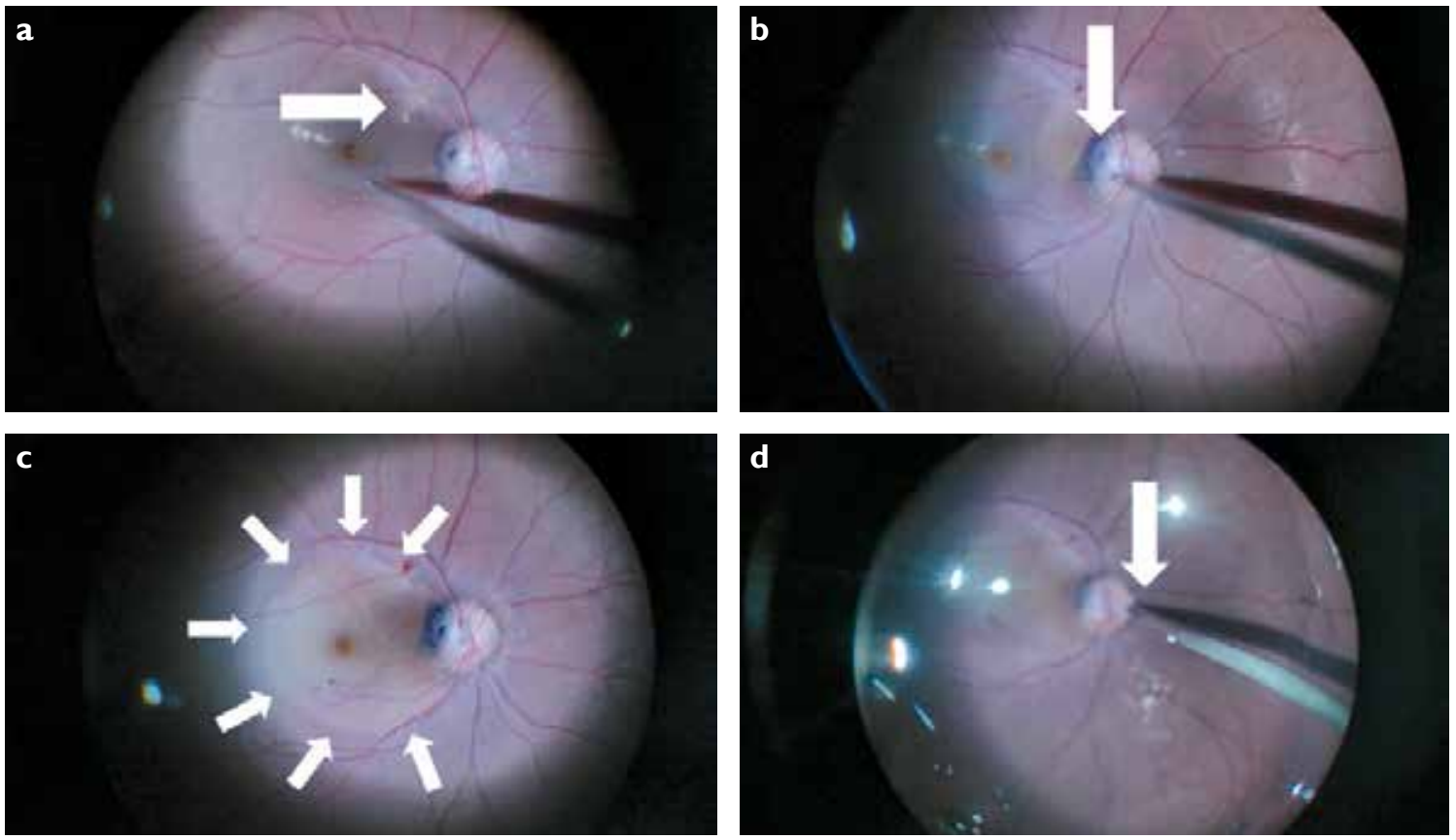

Figure I. (a) The first puncture of internal limiting membrane (ILM) (white arrow) near the optic pit, (b) placing the prepared ILM flap (white arrow) near the optic pit, (c) the borders of the peeled ILM (white arrows), (d) placing the flute needle at the nasal margin of the optic disc in order to passively insert the ILM flap into optic pit cavity.

sufficient to insert it into the pit cavity. Fluid-air exchange was performed again. To insert ILM flap into the optic pit cavity, flute cannula was placed nasally to optic nerve head, and after aspirating last drops of fluid, ILM flap was passively inserted into the optic pit cavity (Figure Id). Surgery was finalized with air-gas exchange with $12 \%$ perfluoropropane. Constellation Vision System (Alcon Laboratories, Inc., Fort Worth, TX, USA) was used for all surgeries. Cut rate was 5000 cuts per minute, vacuum level was $500 \mathrm{mmHg}$, preset intraocular pressure was $21 \mathrm{mmHg}$, and device was in core duty cycle mode during all steps of surgeries. Patients were asked to maintain face-down position for 5 days.

\section{Results}

Six eyes of 6 consecutive patients were operated on using this technique between January and August 2016 by a single surgeon (AO). Three patients (50\%) were female, and 3 $(50 \%)$ were male. Median age of the patients was 25 years (range: 12-45 years). One patient exhibited full-thickness macular hole at first postoperative week (Figure 2b); howev-
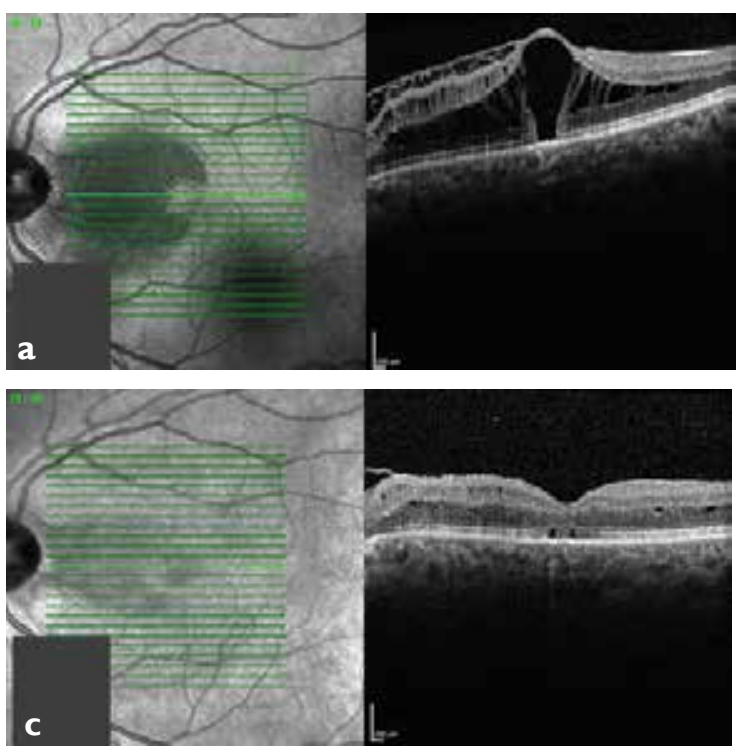

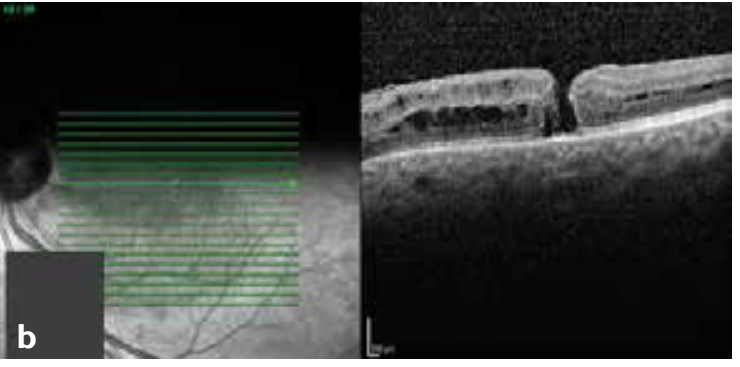

Figure 2. (a) The preoperative optical coherence tomography scan of the patient illustrating very thin retinal tissue on top of an outer macular hole, (b) the full-thickness macular hole on postoperative seventh day, (c) full-thickness macular hole was completely closed after 7 additional days in face-down position. 

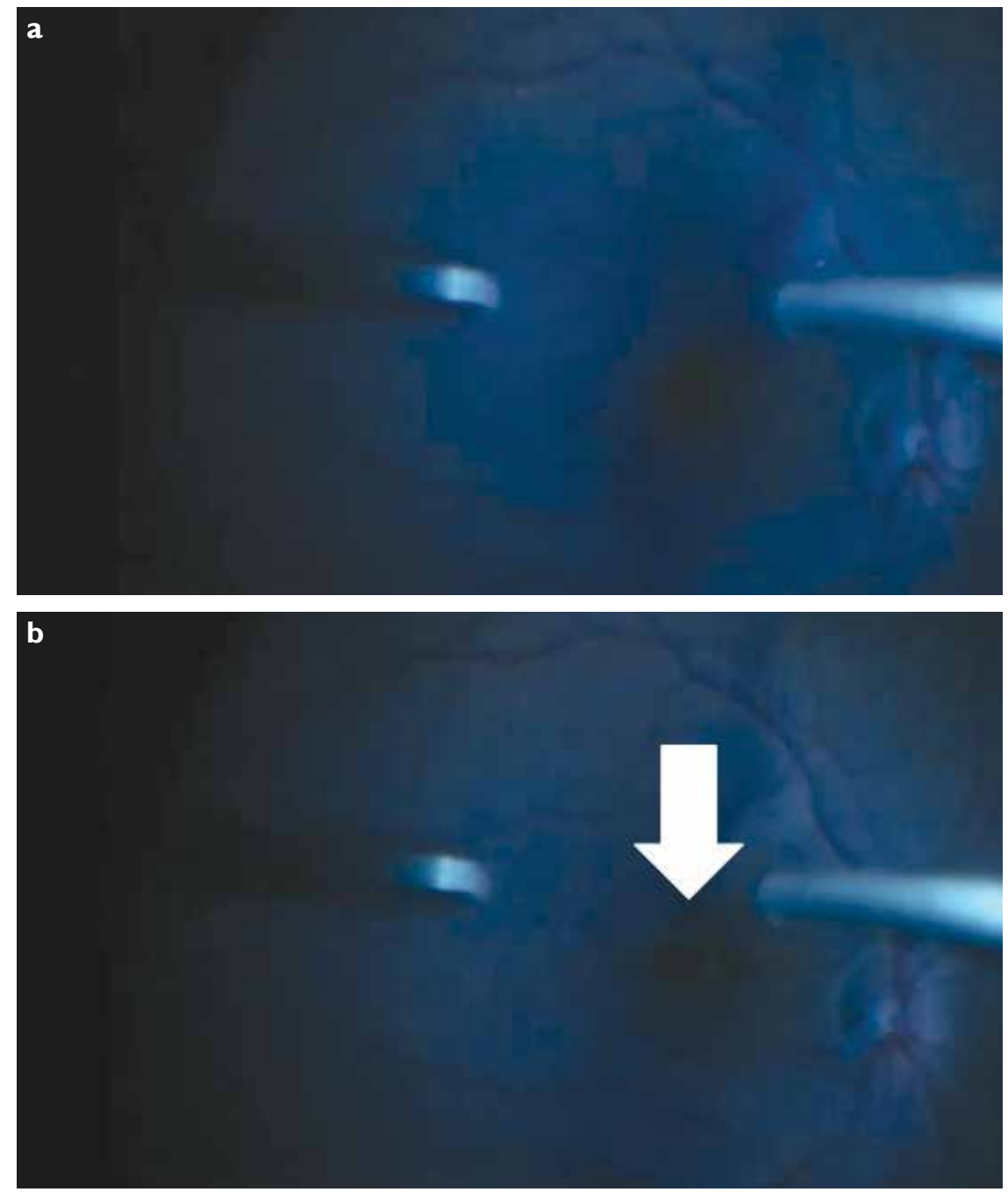

Figure 3. (a) A peroperative image from the operation video while washing out remaining brilliant blue $\mathrm{G}$ after air-fluid exchange. Please note the position of the vitreous cutter. It is very close to the fovea, (b) there is a contrast change at the fovea, a yellow ring encircles a darker area (white arrow), which is thought to be the moment of formation of the full-thickness macular hole.

er, macular hole was fully closed after face-down positioning for 7 days (Figure 2c). When we reviewed our records, we realized that the patient had outer macular hole with very thin retinal layer (Figure 2a). While performing fluid aspiration with vitreous cutter after staining ILM with Brilliant Blue $G$, the surgeon was holding the vitreous cutter very close to the fovea and using a high rate of suction when the thin retinal layer suddenly came up. It was concluded that most probably the full-thickness hole occurred at that moment (Figure 3a, b). No other peroperative or postoperative complications were observed.

\section{Discussion}

In ODPs complicated with OPM, loss of visual acuity is a common feature and usually the prognosis is poor without treatment. Currently there is no standard treatment for ODP. Pars plana vitrectomy alone or in combination with several other procedures seems to be effective with regard to visual and anatomical outcomes. Avci et al., reported successful outcomes with PPV, posterior hyaloid removal, endolaser photocoagulation, and perfluoropropane tamponade (4). Karacorlu et al. described a novel technique that combined PPV with radial optic neurotomy (5). They reported that $86 \%$ of the eyes ( 6 of 7 eyes) had improved vision after surgery. Ozdek et al. used autologous fibrin and gas tamponade for persistent OPM patients and reported successful anatomical outcomes in 2 patients (6).

We know from the literature that PPV alone is an effective method of treatment for OPM. However, it seems that sometimes adjuvant techniques to PPV are needed for 
better outcomes. Reverse ILM flap technique as an adjuvant to PPV was used for persistent macular hole surgery in some studies and was shown to be beneficial. Optic pit is an anatomical defect in the optic nerve, a tissue neighboring the retina. Therefore, a reverse ILM flap might also be beneficial in OPM. Thus, in presently reported technique we combined ILM peeling with reverse ILM flap and omitted laser photocoagulation. This technique may hasten healing process and prevent persistence of OPM; however, further studies are required to verify this hypothesis.

\section{Disclosures}

Financial Disclosure: This surgical technique report was not supported by any company. None of the authors has financial or proprietary interests in any material or method mentioned. These data have not been previously published.

Peer-review: Externally peer-reviewed.

Authorship Contributions: Design and conduct of the study AO, GE; preparation and review - AO, GE; data collection - AO; statistical analysis - (not applicable).

\section{References}

I. Ozkaya A, Alkin Z, Taylan AT, Demirok A. Ocular imaging findings of bilateral optic disc pit in a child. Nepal J Ophthalmol 2013;5:258-6I. Erossre

2. Georgalas I, Ladas I, Georgopoulos G, Petrou P. Optic disc pit: a review. Graefes Arch Clin Exp Ophthalmol 201 I;249: I I I3-22.

3. Kumar A, Gogia V, Nagpal R, Roy S, Gupta S. Minimal gauge vitrectomy for optic disc pit maculopathy: Our results. Indian J Ophthalmol 2015;63:924-6. Crossret

4. Avci R, Yilmaz S, Inan UU, Kaderli B, Kurt M, Yalcinbayir O, et al. Long-term outcomes of pars plana vitrectomy without internal limiting membrane peeling for optic disc pit maculopathy. Eye (Lond) 2013;27:1359-67. Crossre

5. Karacorlu M, Sayman Muslubas I, Hocaoglu M, Ozdemir H, Arf S, Uysal O. Long-term outcomes of radial optıc neurotomy for management of optıc disk pit maculopathy. Retina 2016. Crossret

6. Ozdek S, Ozdemir HB. A new technıque with autologous fibrın for the treatment of persistent optic pit maculopathy. Retin Cases Brief Rep 2016. 